Präv Gesundheitsf 2020 · 15:209-217

https://doi.org/10.1007/s11553-020-00783-z

Eingegangen: 4. April 2020

Angenommen: 7. April 2020

Online publiziert: 8 . April 2020

(c) Springer-Verlag GmbH Germany, part of Springer Nature 2020

\section{Einführung}

Seit der zweiten Märzwoche traten in Deutschland weitreichende Maßnahmen zur Reduktion der Sozialkontakte in Kraft, um die weitere Übertragung des SARS-Coronavirus-2 abzubremsen. Neben den grundlegenden Anordnungen von Isolation (von nachgewiesen positiv Getesteten), von Quarantäne (von Kontaktpersonen ersten und mitunter zweiten Grades) und der Abschottung der vulnerablen Personen wurden weitere drastische Maßnahmen ergriffen. Diese sind u.a. die Schließung der Kindertagesstätten, Schulen und Universitäten. Alleinig Gruppen von bis zu 5 Kindern und kleine Gruppen an Schülern von Eltern aus der sogenannten kritischen Infrastruktur (KRITIS), die keine eigene Betreuung ihrer Kinder sicherstellen können, werden in den offiziellen Strukturen betreut. Des Weiteren wurde das öffentliche Leben über sogenannte Allgemeinverfügungen sukzessive gedrosselt, bis hin zu Ausgangssperren in manchen Bundesländern und besonders betroffenen Gemeinden und Städten. Die Auswirkungen auf die Geschäftswelt, den Mittelstand und die Wirtschaft an sich werden hinlänglich thematisiert, und die finanziellen Opfer sind bisweilen erheblich.

Die Wirkung dieser Art Vollbremsung, die in der Nachkriegsgeschichte bisher einmalig ist, macht sich bereits bemerkbar, und die Neu-Infektionszahlen nehmen nur noch langsamer zu. Sollte sich diese Wirksamkeit als nachhaltig und tatsächlich bestätigen, dann gilt es, jetzt Überlegungen für die nächsten Stufen und den Rest zumindest von 2020,

Josef Weigl

Gesundheitsamt Plön, Schleswig-Holstein, Plön, Deutschland

\title{
Mit Immanuel Kant in die zweite Welle der gegenwärtigen Pandemie
}

bis hin zur 2. Welle und auch darüber hinaus, anzustellen, um jetzt, quasi flussaufwärts, die richtigen Entscheidungen zu treffen. Bundesminister Jens Spahn und der Leiter des Robert Koch-Institut, Prof. Lothar Wieler, haben bereits Signale in diese Richtung gesendet. Die Pandemie fordert die Erkenntnistheorie und die Erkenntnissicherheit in einer ganz besonderen Weise und in einem ernsten Maße heraus.

\section{Methode}

Anhand zentraler Inhalte der Philosophie von Immanuel Kant (1724-1804) soll die anstehende Phase nach den ersten Erfahrungen mit SARS-CoV-2/COVID19 in Deutschland und dem ersten Gipfel der ersten Welle analysiert und beurteilt werden $[14,16]$, was jetzt $\mathrm{zu}$ tun ist und worauf es in den nächsten Monaten, gegebenenfalls mindestens 2-3 Jahren, ankommen wird. Nach den zentralen Fragen Kants soll diese Analyse vorgenommen werden:

Was kann ich wissen?

Was soll ich tun?

Was darf ich hoffen?

Was ist der Mensch?

„Ich“ wäre hier gleichzusetzen mit Deutschland.

\section{Ergebnisse}

\section{Was kann ich wissen?}

\section{Erfahrungen in anderen Ländern}

Hier sind vor allem die frühen Erfahrungen und Daten aus China zu nennen, denn China ist dem Geschehen bei uns um zwei Monate voraus [12]. Da- zu kommen zunehmend die Erfahrungen und die Mitteilungen aus Italien und aus Frankreich. Bisher machen die Länder ähnliche Erfahrungen, und die epidemiologischen Daten gleichen sich. Der Hauptunterschied zwischen den Ländern und den Regionen ist alleinig die Verbreitungsgeschwindigkeit des Virus und das Überfluten der einzelnen Systeme, so dass in vielen Regionen in Italien, Spanien und dem Elsass die Durchseuchung sicherlich weiter fortgeschritten ist als hierzulande, aber eben auch zu einem sehr hohen Preis. Bei dem schnellen Erkennen der Lage und dem Implementieren von Gegenmaßnahmen gab es allerdings bisher Unterschiede. So haben Hong Kong und Singapur sehr schnell gehandelt. Die Erfahrung aus der SARS-Krise 2002/2003 tat da das Seinige dazu $[1,2]$.

\section{Epidemiologie und Modellierungen}

Soeben sind mehrere Modellierungen publiziert worden $[1,3,9,18]$, wobei die Arbeit des Imperial College die für die Betrachtung hier aufschlussreichste ist [9]. Neil Ferguson und Mitarbeiter beschreiben darin für Daten aus Großbritannien, wie groß die Effekte verschiedener nicht-pharmazeutischer Interventionen (NPI) auf die Belastung des National Health Service (NHS) und der Intensivbetten in einer ersten pandemischen Welle wären und zu welchen Verschiebungen in die 2. Welle es unter bestimmten Voraussetzungen käme (• Abb. 1). Die stärksten Effekte auf eine Reduktion der momentanen Belastung des NHS sind von den einschneidenden, generellen Maßnahmen zur Kontaktreduktion $\mathrm{zu}$ erwarten. Diese werden 


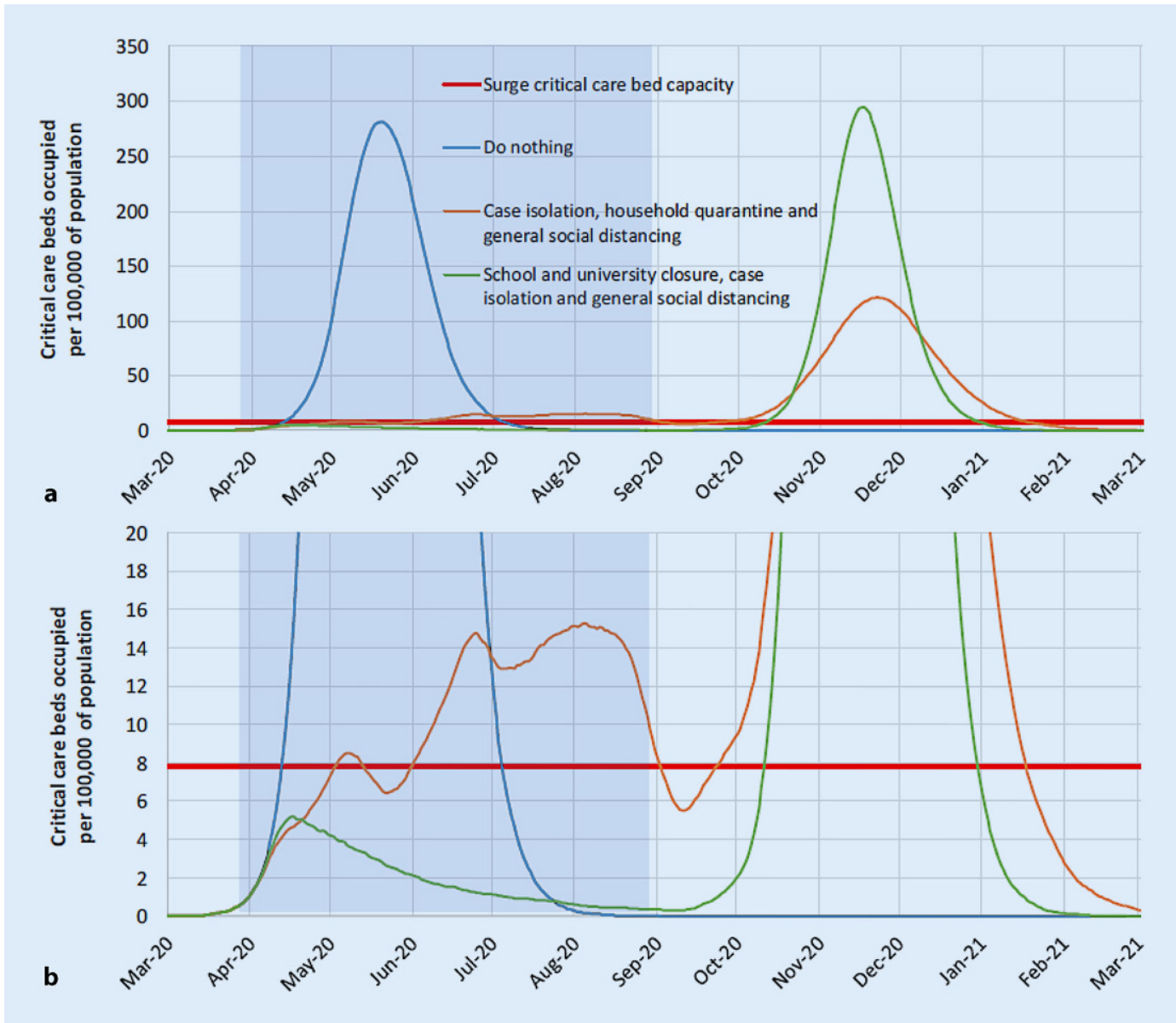

Abb. $1<$ Suppressionsstrategien in Großbritannien anhand der Auslastung der Intensivbettenkapazität (Betten pro 100.000 Einwohner) über die Zeit nach Ferguson et al. [9] (Erlaubnis zum Abdruck vom Erstautor erhalten am 1. April 2020). Surge critical care capacity $=$ derzeitige Intensivbettenkapazität (in Großbritannien bisher 8 pro 100.000 Einwohner, in Deutschland 34 pro 100.000 vor einer Aufstockung)

in ihrer Langzeitwirkung mit und ohne gleichzeitige Schließung der Kitas, Schulen und Universtäten betrachtet. Beides zusammen über eine längere Dauer würde zu einer nicht verkraftbaren 2. Welle ab November 2020 führen, sodass die Autoren weitere Betrachtungen anstellten. Anhand der Belastung der Intensivstationen (ICUs) modellierten sie Lockerungen der Maßnahmen, um bei möglichst Nicht-Überbelastung des NHS eine möglichst umfassende Durchseuchung der Allgemeinbevölkerung, ohne die vulnerablen Gruppen, zu erreichen. Die Modellierung ergab intervallartige, pulsierende Lockerungen mit jeweils einem verzögerten Wiederanstieg der ICU-Bettenbelastung (• Abb. 2). Die Größe der identifizierten Triggerpunkte (sprich Belegung der ICU Betten im NHS) für Lockerungen bzw. Verschärfungen der Maßnahmen könnten für Deutschland sicherlich größer sein aufgrund der hierzulande deutlich höheren Verfügbarkeit von Intensivbetten.
In Großbritannien kommen auf 100.000 Einwohner 8 Intensivbetten, in Deutschland ohne akute Aufstockung bereits 34 . Mit einer Verdoppelung der Kapazitäten binnen weniger Wochen wird gerechnet. Nach Berechnung von Ferguson et al. wären zwei Drittel der verbleibenden Zeit des Jahres 2020 die Maßnahmen „an“. Dies könnte durch die größeren Intensivbettenzahlen in Deutschland weniger werden.

\section{Prinzipien der Impfstoff- entwicklung}

Warum können wir nicht erwarten, noch vor der 2. Welle einen ausreichend getesteten Impfstoff zur Verfügung zu haben? An dieser Stelle ist es dienlich, nochmals an die etablierten und herausragenden Schritte der Impfstoffentwicklung $\mathrm{zu}$ erinnern. Vorbedingungen sind Impfstoffplattformen, die eine Entwicklung eines Impfstoffes gegen SARS-CoV-2 aussichtsreich erscheinen lassen. Das Haupttarget bzw. Antigen ist das Spike-
Protein, das Andockprotein von SARSCoV-2 an den Rezeptor der Wirtszelle und zwar in der Präfusionsform [21]. Es gibt über die Jahre mehrere solcher Plattformen von RNA/DNA Ansätzen, über Vektoren-basierte bis hin zu den klassischen Plattformen mit rekombinanten Proteinen oder (meist abgetöteten) Viruspartikeln mit oder ohne Adjuvantien. „Dose-sparing“ wäre in Anbetracht des riesigen Bedarfs an einem Impfstoff gegen COVID-19 ein wichtiger Aspekt, wie auch bei pandemischen Influenzaimpfstoffen. Von besonderem Wert sind solche Plattformen, die bereits die nötigen Produktionsstätten und Produktionskapazitäten belegt haben, denn die Entwicklung eines Impfstoffes ist das eine, eine GMP konforme Massenproduktion das andere. GMP steht für "good manufacturing practice“.

Nach der Herstellung der meist molekularbiologischen oder stereo-/strukturchemischen Konstrukte folgen die toxikologische Prüfung und dann die 
Auswahl der verheißungsvollsten Kandidaten für die präklinische Entwicklung. Nach den einfachen Spezies wie Mäusen unterschiedlicher immunologischer Prägung erfolgen weitere Testungen in Tierspezies, die für den betreffenden Erreger besonders geeignet und prädiktiv sind. Bei ARI-Erregern ist das meist das Frettchen. ARI steht für akute respiratorische Infektion. Sequentiell oder auch bereits parallel dazu müssen mindestens zwei Primaten (Makaken) nach erfolgter Impfung mit dem Wildvirus infiziert/ provoziert werden, um ein sogenanntes „Immune-enhancement", wie es bei Ortho- und Paramyxoviren beschrieben ist, auszuschließen. Bei Nidavirales, $\mathrm{zu}$ denen SARS-CoV-2 gehört, ist das bisher weniger umfangreich untersucht. Allerdings ist die Beobachtung einer fokalen Hepatitis bei Frettchen nach Impfung mit einem MVA-SARS-SpikeKandidaten und Provokation mit dem Wildvirus beunruhigend [5].

Sollten diese Untersuchungen alle erfolgreich und ohne besorgniserregendes „safety“-Signal durchgeführt worden sein, kommt der Schritt in die klinische Entwicklung, nachdem auch die immunologischen Nachweisverfahren etabliert wurden. Allen voran steht dabei der neutralisierende Antikörpertest, ein funktionaler Test. Je nach Vorerfahrungen mit der entsprechenden Impfstoffplattform würden dann in einer Phase 1 Eindosis- und ZweidosisGaben untersucht werden, und das bei Männern und Frauen, denn Geschlechtsunterschiede in der Verträglichkeit sind eine zentrale Frage der Phase 1 neben der grundsätzlichen Verträglichkeit. Die ersten Patienten würden stufenweise in die Studie aufgenommen und geimpft werden („staggered enrolment“). Nach einer Nachverfolgung von mindestens sechs Wochen würden die entsprechenden safety-Parameter sowohl klinisch als auch laborchemisch („reactogeniciy“) und die ersten immunologischen Daten („immunogenicity“) erhoben. Frühestens nach Kenntnis dieser Daten könnte eine Phase 2a mit einer Fallzahl von wenigen Hundert Personen zur Dosisfindung, Immunogenität und zur Festlegung des Impfregimes erfolgen. Dies würde zudem die safety-

Präv Gesundheitsf 2020 · 15:209-217 https://doi.org/10.1007/s11553-020-00783-z

(c) Springer-Verlag GmbH Germany, part of Springer Nature 2020

\section{J. Weigl \\ Mit Immanuel Kant in die zweite Welle der gegenwärtigen Pandemie}

\section{Zusammenfassung}

Hintergrund. Die Wirkung der größten Einschränkung der Bewegungsfreiheit der deutschen Bürger seit Ende des Zweiten Weltkrieges und der Ölkrise 1973 als beabsichtigte Bremswirkung auf die 1. Welle der Pandemie wird soeben erwartet. Die Planungen zum weiteren Vorgehen für die kommenden Wochen und Monate müssen jetzt angestrengt werden.

Methode. Unter Zuhilfenahme der Philosophie von Kant sollen die Überlegungen und Planungen geordnet werden.

Ergebnisse. Die Modellierung der Forscher des Imperial College, London, lassen eine übermächtige 2. Welle für den Herbst/Winter 2020 erwarten, wenn die jetzigen Maßnahmen zu lange aufrechterhalten werden. Eine baldige Lockerung der Maßnahmen ist angezeigt, um eine fraktionierte Durchseuchung der Bevölkerung, ohne konsekutive Überlastung der Versorgungsstrukturen, allen voran der Verfügbarkeit von Beatmungsplätzen, noch vor der nächsten
Influenzasaison 2020/2021 zu ermöglichen. Die Aussicht auf einen allenfalls marginal vor der Zulassung geprüften Impfstoff vor Beginn der 2. Welle, der dann höchstens für Menschen mit einem sowieso hohen Risiko bei SARS-CoV-2 in Frage käme, sollte jetzt zu keinen Verzögerungen bei den auszudifferenzierenden, intermittierenden Lockerungen führen.

Schlussfolgerungen. Weitere, noch detailliertere und voneinander unabhängige Modellierungen auf der Basis deutscher Daten sind dringend notwendig. Ein über die Hierarchien hinweg heterogenes Gremium müsste darauf basierend die differentiellen Lockerungen diskutieren und in Anbetracht von erheblichen Restunsicherheiten beschließen.

Schlüsselwörter Durchseuchung · Impfstoffentwicklung . Lockerungen · Nicht-pharmazeutische Intervention · Phase-3-innere Einstellung

\section{With Immanuel Kant into the second wave of the current pandemic}

\section{Abstract}

Background. The efficacy of the most serious limitation of free movement of the German citizens since the end of the World War II and the Oil Crisis 1973 as intended countermeasure against the first wave of the pandemic is currently expected. The planning in regard to further action for the coming weeks and months has to take place now. Methods. With the help of the principles of the German philosopher Kant, the thinking and planning is framed.

Results. The modelling by the researchers of the Imperial College, London, make an oversized second wave in autumn/winter 2020 most likely, if the current countermeasures are kept in place for too long. A timely relaxation of the measures is warranted to allow a staggered attack by the virus onto the population (German: "Durchseuchung") before the next influenza season 2020/2021 without overburdening the health care infrastructure especially the respiratory care bed capacity. The tempting perspective of a vaccine available before the second wave in autumn, should not lead to any delay in the intermittent relaxation of the countermeasures. A pre-licensure marginally tested vaccine would only be eligible for people with an anyway high risk in regard to SARS-CoV-2.

Conclusions. Further, more detailed and from each other independent models on the basis of data from Germany are urgently needed. Upon these a heterogeneous transhierarchical committee would need to discuss and make decisions in regard to differentiated relaxations of measures given the high remaining uncertainties.

\section{Keywords}

Durchseuchung · Relaxation · Non-pharmaceutical interventions - Phase-3-habit . Vaccine development 


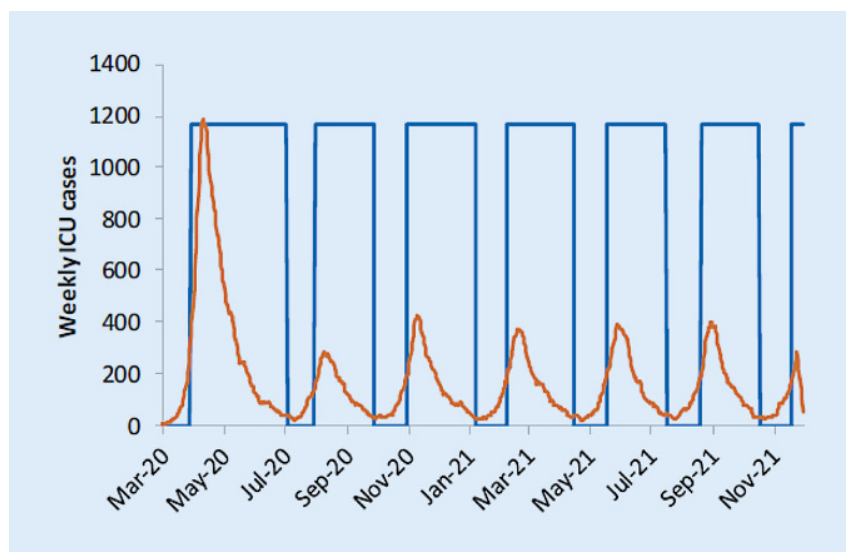

Abb. 2 A Angepasstes Triggern der Maßnahmen bei Belegung von 100 Intensivbetten und deren Lockerungen bei 50 Intensivbetten (absolute Zahlen). Die erwogenen Maßnahmen sind die Allgemeinempfehlung zur Kontaktreduktion und die Schließung von Kitas, Schulen und Universitäten in Großbritannien nach Ferguson et al. [9]. (Erlaubnis zum Abdruck vom Erstautor erhalten am 1. April 2020)

Datenbank auch schon etwas vergröBern. Wiederum nach entsprechendem Zeitfenster, und da sind die Entwicklungspläne („Gantt charts") knallhart, könnte man nach einer Phase $2 b$ - oder einer kleinen Phase 3a-Studie den Impfstoff unter Auflagen zulassen. „Gantt charts" sind das zeitliche Planungsgerüst einer Produktentwicklung mit untereinander zeitabhängig verknüpften Arbeits- und Entwicklungsabschnitten. Die Zeit kann sich keiner erkaufen und die Zeit ist auch in der Impfstoffentwicklung die härteste Variable, auch wenn mehrere Abkürzungen versucht werden. Eine experimentelle Phase $2 b$ Studie mit Exposition der Impflinge mit dem Wildvirus wäre schwer bei einer Ethikkommission durchzubekommen, da es bisher keine „rescue“-Therapie in Anbetracht mangelnder Therapeutika gibt. $\mathrm{Ob}$ das Argument jüngerer Impflinge in einer Phase $2 b$ eine Lösung wäre, ist in Sachen ethischer Beurteilung schwer vorherzusagen. Für eine zügige Phase $3 a$ Wirksamkeitsstudie könnte man ein cluster-randomisiertes Verfahren in einem hochprävalenten Ausbruchsgebiet anstreben, um schnell ein Divergieren der Verumgruppe von der Kontrollgruppe beobachten zu können. Wegen des Zeitdrucks sind aber einfachere Designs vor der Schnellzulassung attraktiver, sodass, wie üblich, eine Cluster-randomisierte Studie als sogenannte Phase $3 \mathrm{~b}$ erst nach der Zulassung durchgeführt werden würde. Eine Phase 3a Studie sollte vor allem unter dem Gesichtspunkt safety mit 10.000 oder mehr Impflingen durchgeführt werden. Ein „enrolment" selbst bei einer derart groBen Anzahl geht bei bereits getroffenen Vorbereitungen binnen $48 \mathrm{~h}$ (persönliche Erfahrung bei GSK). Der erste „safety read out" nach 30 Tagen könnte dann der Zeitpunkt für eine Marktfreigabe, sprich Notfalllizensierung, sein, und die Wirksamkeitsendpunkte und die mindestens 6-Monate safety-Endpunkte könnten über die Marktfreigabe („marketing authorisation") hinaus weiterverfolgt werden. Das gesamte klinische Entwicklungsprogramm müsste selbstverständlich von einem DSMB („data safety monitoring board“) begleitet werden und klare Stopsignale vereinbart werden. Wäre die Not besonders groß, dann würde, vermutlich in den USA, eine noch deutlichere Abkürzung durch die FDA („Food and Drug Administration") unter dem Druck der US-Regierung erwogen, zum Beispiel nach der Phase 2, um zumindest Risikogruppen mit dem nur teilweise geprüften Impfstoff $\mathrm{zu}$ injizieren.

Wichtig bei all dem ist zu betonen, dass die gesamte Impfstoffentwicklung selbst unter pandemischen Bedingungen erfolgen würde. Das heißt, dass die Studiengruppen durch Interferenz durch das Wildvirus verzerrt oder sogar durcheinander gewirbelt werden könnten, so denn die Impflinge der Phase 1 und 2 nicht mindesten 6 Wochen unter Qua- rantäne blieben. Wirksamkeitsstudien würden durch NPI gegebenenfalls in die Länge gezogen werden, weil es zu weniger Infektionen kommen würde. Des Weiteren könnten auch die Mitarbeiter der Firmen und der CRO („Contract Research Organization") erkranken und ausfallen, was wiederum zu Verzögerungen in den Studien und ihrer Ergebnisse führen könnte.

\section{Was soll ich tun?}

Wir sollen so vorgehen, als ob ein Impfstoff gar nicht käme, und jetzt die Zeit umgehend nutzen, die Population dosiert durchseuchen zu lassen und Nebenwirkungen, sprich schwere Verläufe, im Krankenhaussystem aufzufangen und die Betten- und Beatmungskapazität vorzuhalten und zu nutzen. Wir sollten die dosierte Durchseuchung bis November 2020 soweit möglich erlauben, um das "Sommerloch“ der Krankenhausbelastung optimal zu nutzen, den "Geist quasi fraktioniert aus der Flasche lassen". Um dies umsetzen zu können, müssen noch viel detailliertere Modellierungen, basierend auf Daten aus Deutschland, durchgeführt werden. Die Modellierung von Ferguson et al. vom 16. März 2020 beruht auf Daten aus Großbritannien und dem dortigen NHS und führte deshalb zu ausgesprochen niedrigen Triggerwerten für eine ICU-Belegung (100 bzw. 50), die in Deutschland mindestens 4 bis $5 \mathrm{Mal}$ höher sein könnten (• Abb. 2). Deshalb sollte das Imperial College umgehend einen Auftrag und die notwendigen Daten aus Deutschland von der Bundesregierung erhalten, um für Deutschland auf der Basis hochauflösender Daten eine Modellierung zur Verfügung zu stellen. Parallel dazu müssten unabhängig davon mindestens zwei weitere Gruppen, auch unabhängig voneinander, Modellierungen durchführen, um die Robustheit der Einflussgrößen unabhängig zwischen Gruppen zu bestätigen, denn es würde sehr viel davon abhängen. „All models are wrong, but they are helpful“ [18], und deshalb bedürften auch derartige Daten der epikritischen Beurteilung eines Expertenkomittees. Dieses Komitee sollte unbedingt über mehrere Hierarchieebenen hinweg zusammengesetzt sein, ent- 
sprechend des sogenannten „post-westphalian approach“ nach Fidler [10]. Nur dann wären der Realität und den operativen Gegebenheiten angemessene Entscheidungen zu erwarten, die ohnehin mit einer Restunsicherheit behaftet wären. Getroffen werden müssten diese Entscheidungen zur Lockerung bzw. Aufhebung der Maßnahmen von Zuständigkeitswegen letztlich vom jeweiligen Bundesland einzeln.

Sollte ein nur begrenzt vorab geprüfter, schnell entwickelter Impfstoff noch in der zweiten Jahreshälfte einsetzbar sein, ungeachtet der nicht gesicherten Massenverfügbarkeit auch in Deutschland, dann käme der Impfstoff in Anbetracht der Erkenntnisse zur „burden of disease“, der Mortalität und Morbidität, vor allem bei vulnerablen Gruppen und höchstens noch bei Funktionsträgern mittleren und höheren Alters und Personen an Schnittstellen, wie zum Beispiel noch nicht immunen Verkäuferinnen in Lebensmittelgeschäften, in Betracht.

\section{Was darf ich hoffen?}

Wir dürfen hoffen, den Hauptteil der Bevölkerung vor einer eventuellen übergroßen 2. Welle immun zu bekommen, bevor der Druck auf die Krankenhäuser saisonal durch andere ARI-Viren, einschließlich Influenzaviren, in der kommenden Wintersaison wieder ansteigen wird. Das wäre das Ziel und die Hoffnung; das heißt, das Abwenden einer übermächtigen 2. Welle (• Abb. 1; [9]). In meiner Modellierung [18] habe ich quasi die ideale Zielvorgabe angenommen; nämlich eine möglichst konstante Anflutung von schwer Erkrankten in das Versorgungssystem über sechs Monate; das heißt von jetzt bis in den Herbst 2020. Die wellenartige Durchseuchung durch Einsetzen und Wegnahme der Trigger laut Ferguson et al. wäre die quasi asymptotische Annäherung an dieses Ideal.

Dass der Infektionsdruck in der wärmeren Jahreszeit nachlassen wird, ist bei einem Erreger, dem gegenüber die gesamte Population bisher als immunologisch naiv angenommen wird, unwahrscheinlich. Ganz im Gegenteil, durch das Abnehmen der Inzidenz der saisonalen Erreger [19] findet SARS-CoV-2 dann leichter seine Opfer, denn die Konkurrenten, sprich saisonale ARI-Viren, ziehen sich zurück. Das heißt, die Konkurrenz um die Opfer nimmt ab und SARSCoV-2 kann zunehmen [20]. Sowohl bei der Spanischen Grippe 1918-1920 als auch bei H1N1 2009 erstreckte sich die erste Welle in den Sommer hinein. Eine wie auch immer geartete kreuzimmune Teilpopulation würde die Höhe der 1. und der 2. Welle dämpfen [3]. Am plausibelsten erscheint diese zurzeit für die jüngeren Altersgruppen, da andere Coronavirus-Infektionen erst kürzer zurücklagen und noch keine Immunoseneszenz eingesetzt hat.

Die Hoffnung auf ein „Magic bullet“ eines Impfstoffes kann zu Wunschdenken verführen. Der Einsatz eines vor der Zulassung sehr limitiert getesteten Impfstoffes kommt nach einer entsprechenden Nutzen-Risiko-Abwägung am ehesten bei Personen mit hohen persönlichen Risiko für einen schweren und komplizierten Verlauf der COVID-19 in Betracht, wie oben bereits dargestellt. Alle derzeitigen Impfstoffkandidaten haben als Antigen das Spike-Protein von SARS-CoV-2, das Oberflächenprotein, das für das Andocken am Rezeptor in den menschlichen Atemwegen dient. Die Beobachtung von Czub et al. [5], dass MVA-Spike-Protein von SARS geimpfte Frettchen nach Provokation mittels Infektion durch das SARS-Wildvirus fokale Lebernekrosen im Vergleich zu nur Wildvirus infizierten Frettchen bekamen, spricht für eine „immunization enhanced disease“, ein Signal, das zur Vorsicht mahnt. Wenn der Impfstoff ein noch relativ unbekanntes Sicherheitsprofil hat mit möglichen Spätfolgen und Langzeitwirkungen, wie bei Pandemrix ${ }^{\circledR}$, einem pandemischen Impfstoff gegen H1N1 2009, und der bedauerlicherweise beschriebenen Häufung von Narkolepsie 2010 [7], dann wäre dies nur in dieser Gruppe vertretbar, die ansonsten eine schlechte Prognose hat. Man könnte sich mit Vergleichen aus der Onkologie rechtfertigen, in der auch Präparate mit deutlich ungünstigerem Sicherheitsprofil in Anbetracht der Schwere der Grundkrankheit akzeptiert werden. Das überraschende Auftreten des GuillainBarré-Syndromes nach einem anderen
Impfstoff gegen ein H1N1-Virus vom Schwein 1976 ist eine lehrreiche Episode eines überschnell eingesetzten und unzureichend getesteten Impfstoffes [8]. Für die Altersgruppen mit einen ausgesprochenen niedrigen Komplikationsrisiko bei COVID-19 käme ein nur begrenzt klinisch getesteter Impfstoff nicht oder nur sehr schwerlich in Frage, auch nicht mit der Absicht, die 2. Welle und folgende Wellen abzubremsen. Dafür müsste das safety-Profil ausgesprochen günstig sein und man auf eine hohe „supportive evidence“ aus der Historie der entsprechenden Impfstoffplattform zurückgreifen können.

\section{Was ist der Mensch?}

"Alles beginnt und endet beim Menschen" (Kant). Wie Joshua Lederberg (1925-2008), amerikanischer Molekularbiologe, bereits in den 1990er Jahren sagte, sind Viren die größten Feinde des Menschen: „Viren sind die einzigen Rivalen um die Herrschaft über unseren Planeten. Wir müssen auf Draht sein, um mit ihnen Schritt zu halten“. Zu welchen Dysruptionen es in unseren hochentwickelten Gesellschaften und verflochtenen Wirtschaftssystemen kommen kann, erleben wir soeben life mit. Nach der Pandemie ist vor der Pandemie, und die Erfahrungen der letzten Jahre mit H1N1 2009/2010, Ebola 2014 und 2019/2020, ZIKA 2015/2016, insbesondere in Brasilien mit dem epidemischen Auftreten von mikrozephalen Kindern, dem Anschwellen der Bedrohung durch das Westnilfiebervirus (WNV) und anderer ARBO-Viren, auch in Deutschland, und jüngst immer mehr Fälle an KongoKrim-Hämorrhagischen Fieber (CCHF) auch in Europa, der von mir „ZeckenEbola“ genannten Erkrankung, zeigen, dass die Bedrohungen durch Infektionskrankheiten auch nach dieser aktuellen Pandemie fortbestehen werden. Selbst die Bedrohung in Form der jetzigen Pandemie durch ein neues Coronavirus war längst vorher bekannt [15].

Für die aktuelle Pandemie sind die jüngeren Altersgruppen bisher weniger im Fokus, dafür aber die älteren und vorbelasteten Menschen, die sogenannten Risikogruppen: das Damocles- 
Tab. 1 Herausforderungen der Impfstoffentwicklung und des Pandemiemanagements

\begin{tabular}{|c|c|c|c|}
\hline Aspekt & Impfstoffentwicklung und Management & Pandemie und Management & Konsequenzen \\
\hline $\begin{array}{l}\text { Themen und } \\
\text { "targets": } \\
\text { VUCA- } \\
\text { technisch } \\
\text { schwierig }\end{array}$ & $\begin{array}{l}\text { Die relativ einfachen „targets“ der Impfstoff- } \\
\text { entwicklung sind bereits entwickelt: die jetzi- } \\
\text { gen targets sind schwieriger: immunologisch, } \\
\text { stereo- \& strukturchemisch usw. }\end{array}$ & $\begin{array}{l}\text { Eine Pandemie ist „VUCA komplex“ und } \\
\text { nicht alle Einflussgrößen können kontrol- } \\
\text { liert werden und bedürfen weitreichender } \\
\text { Einschnitte }\end{array}$ & $\begin{array}{l}\text { Die „success probability“ ist limi- } \\
\text { tiert bis sehr anfällig }\end{array}$ \\
\hline $\begin{array}{l}\text { Regulatori- } \\
\text { sches }\end{array}$ & $\begin{array}{l}\text { Die Behörden FDA, EMA und PEI haben in pan- } \\
\text { demischen Fragen seit der Mitte der 2000s da- } \\
\text { zugelernt. Dennoch sind Impfstoffentwicklungs- } \\
\text { programme sehr teuer und können durch regu- } \\
\text { latorische Auflagen bisweilen nur noch von den } \\
\text { großen Firmen gestemmt werden, es sei denn, } \\
\text { die öffentliche Hand (BMBF) oder PPP (BMGF } \\
\text { oder CEPI) greifen unter die Arme }\end{array}$ & $\begin{array}{l}\text { Die Verwaltungsbehörden einschließ- } \\
\text { lich Ministerien und Kreisverwaltungen } \\
\text { sind gefragt, via schnellen und eingrei- } \\
\text { fenden Verfügungen die notwendige } \\
\text { Kontaktreduktion im Rahmen der NPI zu } \\
\text { veranlassen und zu kontrollieren }\end{array}$ & $\begin{array}{l}\text { Flexibilität der Behörden ist zentral } \\
\text { unter Berücksichtigung der do- } \\
\text { minierenden Einflussgrößen und } \\
\text { deren Modifikation. Die Verhältnis- } \\
\text { mäßigkeit und Mindestsorgfältig- } \\
\text { keit sind zu wahren }\end{array}$ \\
\hline $\begin{array}{l}\text { Führung und } \\
\text { Management }\end{array}$ & $\begin{array}{l}\text { Die Herausforderungen der Impfstoffentwicklung } \\
\text { heute sind erheblich und in ihrer Komplexität } \\
\text { groß. Die großen Firmen verbürokratisieren und } \\
\text { die kleinen haben ein hohes Risiko, dass sie bei } \\
\text { Fehlschlägen nicht überleben. } \\
\text { Der „executive warfar" [6] ist der Impfstoffent- } \\
\text { wicklung hinderlich und lässt das System biswei- } \\
\text { len hinter seinen Möglichkeiten zurück stehen }\end{array}$ & $\begin{array}{l}\text { Die Herausforderungen einer Pandemie } \\
\text { sind hochspezifisch und einmalig, so dass } \\
\text { fortlaufend Entscheidungen notwendig } \\
\text { werden, die nicht in alle Richtungen ab- } \\
\text { gesichert sein können, sowohl von den } \\
\text { Daten als auch von der Erfahrung und } \\
\text { zuletzt auch vom Zeitdruck her }\end{array}$ & $\begin{array}{l}\text { Führungs- und Entscheidungs- } \\
\text { schwächen ganz oben haben gro- } \\
\text { ße Unwucht flussabwärts zur Folge. } \\
\text { Kants Maxime: „was wir gemacht } \\
\text { haben, können wir auch wieder } \\
\text { ändern“ [16] tut not. } \\
\text { VUCAplus ist aktueller denn je [17] }\end{array}$ \\
\hline \multicolumn{4}{|c|}{$\begin{array}{l}\text { BMBF Bundesministerium für Bildung und Forschung, BMGF Bill and Melinda Gates Foundation (Seattle), CEPI Coalition for Epidemic Preparedness } \\
\text { Innovations (London, Oslo), EMA European Medical Agency (Amsterdam), FDA Food and Drug Administration (US Zulassungsbehörde, Washington D.C.), } \\
\text { NPI nicht-pharmazeutische Interventionen, PEI Paul-Ehrlich-Institut (Langen), PPP Public Private Partnership, VUCA volatile, uncertain, complex, ambiguous } \\
\text { (volatil, ungewiss, komplex, uneindeutig) }\end{array}$} \\
\hline
\end{tabular}

Schwert für die Altenpflegeheime bleibt über den Fortgang der Pandemie bestehen, ganz unabhängig davon, welche Lockerungsmaßnahmen generell oder intermittierend getroffen werden. Für die meisten Einrichtungen bleibt es nur eine Frage der Zeit, bis es zu "Einschlägen“ kommen wird.

\section{Management in der Impfstoff- entwicklung im Vergleich zum Pandemiemanagement ( $\bullet$ Tab. 1)}

Beide stehen vor der gleichen Herausforderung wie die Erkenntnistheorie an sich: „Möglichst sichere Erkenntnisse erzielen und dazu Verknüpfungen bilden und Synergien herstellen“.,Neues Wissen und freies Denken schaffen Wertschöpfung, auf die zu verzichten sich kein Unternehmen leisten sollte" [16].

\section{"Phase 3 habit", sprich innere Einstellung}

Eine der Hauptaufgaben der Gesundheitsämter in der Pandemie ist es, die verschiedensten Stakeholder in die Spur zu bringen, denn nur allzu sehr haben auch viele Führungskräfte und Ärzte samt Ärzteorganisationen eigenes, proaktives, der Gesamtherausforderung angemes- senes Verhalten und Arbeitseinstellung weitgehend verlernt. Die „Phase 3 innere Einstellung" mit Betrachtung des Gesamten und vor allem des nächsten Schrittes aus der Sicht des Nächsten, sodass dieser optimal weiterarbeiten kann, ist die zentrale Einstellung, auf die es ankommt. Oft muss ich das Gleichnis von dem Wurf junger Welpen bemühen, bei dem der, der sich zuerst auf den Rücken legt und sich ergibt, meint, die meiste Hilfe zu bekommen. Dieses Verhalten ist in Anbetracht der Herausforderung einer Pandemie inakzeptabel. "Aufklärung ist der Herausgang aus der selbstverschuldeten Unmündigkeit. Unmündigkeit ist das Unvermögen, sich seines Verstandes ohne Leitung eines anderen zu bedienen“ (Kant). Dieser Satz ist in der Pandemie aktueller denn je.

Des Weiteren ist die Absicherung allen Tuns auf den oberen Etagen zugunsten des vertrauensvollen Überlassens an nachgeordnete Strukturen wichtig. Das Bewusstsein, dass in einer VUCA (,volatile, uncertain, complex, ambiguous") dominierten Situation auch Fehlentscheidungen möglich sind, ist wichtig, sowohl für die Entscheider, als auch für die
Betroffenen bzw. die Bevölkerung, die ein Mindestmaß an Toleranz aufbringen müssen [17]. Allerdings ist an die Adresse der Führungsebenen mit Kant zu sagen: „Was wir gemacht haben, können wir auch wieder ändern“. Manche Änderungen, wenn sie sich als widrig erweisen oder über das Ziel hinausschießen, sollten schnell wieder zurückgenommen werden, um das Vertrauen der Bürger nicht zu verlieren. Das wichtigste Gut, das wir im Zusammenspiel haben, ist das Vertrauen auf allen Ebenen, bis hin zur Bevölkerung, „the speed of trust" [4]. ,Es kommt auf jeden an" (Zitat des Autors, Ostholsteiner Anzeiger vom 28. Februar 2020).

Hilfreich wäre, wenn endlich alle Ebenen von den Regierenden bis zu den Ärzteorganisationen in die Phase-3-Einstellung finden würden. Besonders hinderlich sind weitreichende Äußerungen von ganz oben, ob vom Ethikrat oder dem Robert Koch-Institut, weil man bei zu apodiktischen Inhalten in der Peripherie allzu große Mühen hat, dagegen anzudiskutieren. 


\section{Diskussion}

Das Bild des Indianerstammes, der aus den Sommerjagdgründen auf mühseligem und langem Weg mit seinen Nahrungsreserven in die Winterquartiere zieht, um zu überleben, könnte hilfreich sein. Alte und schwache Stammesmitglieder sondern sich dann ab und bleiben zurück, um den Marsch zum Fortbestand des Stammes nicht zu gefährden. Um das Bild möglichst zu vermeiden, ist die 2. Welle möglichst niedrig $\mathrm{zu}$ halten. Da das Risiko für die vulnerablen Gruppen nicht weggehen wird, bis SARS-CoV-2 zu einem gewöhnlichen, endemischen, ARI-Erreger wird, wie bereits andere Coronaviren, sollten sich die Entscheidungen doch mehr und mehr am volkswirtschaftlichen Fortgang der Gesellschaft orientieren.

Des Weiteren stellen sich Fragen wie:

- Wie viel verkraftet unsere Bevöl-

kerung, sprich wie hoch ist ihre

Resilienz, über einen längeren Zeit-

raum unter den Restriktionen $\mathrm{zu}$ leben?

- Wie viel verkraftet die Wirtschaft und die Finanzen?

- Wie kann die Nahrungsgrundlage sichergestellt werden?

- Werden die Spannungen und Konflikte innerhalb und außerhalb

Deutschlands zunehmen?

Ganz schnell ist man da bei den apokalyptischen Reitern von Dürer: Seuchen, Krieg, Wirtschaftskrise und Hungersnot, die miteinander verwoben sind. Es ist immer zu Fragen, wie sehen alternative Szenarien aus und wie realistisch und $\mathrm{zu}$ welchen Preis können diese erreicht werden. Keines wird zum Nulltarif zu haben sein.

\section{Was kann ich wissen?}

Beziehungsweise, was wissen wir noch nicht?

Momentan ist unklar, wie die Seroepidemiologie in der deutschen Bevölkerung aussieht. Das heißt, welcher Prozentsatz ist wirklich für SARS-CoV-2 anfällig und wie viel haben aus bisher unbekannten Gründen eine Kreuzimmunität, wie zum Beispiel jünger zurückliegende
Infektionen mit den endemischen Coronaviren, allen voran den $\beta$-Coronaviren wie z. B. OC43 [19]. Die Kreuzimmunität würde entsprechend zur „herd immunity threshold" [18] beitragen und weniger Erkrankungen wären zu erwarten [3]. Auch die Frage nach einer „antigenic-sin“ durch frühere Infektionen, wie bei Influenza bekannt, wird wissenschaftlich in Sachen Morbidität relevant werden.

Wie schnell wird Deutschland eine ausreichende Versorgung mit qualitativ gesicherter Schutzausrüstung sicherstellen können? Erweist sich eine der bereits als Therapeutika getesteten Substanzen als wirksam? Das würde auch Entscheidungen für Phase $2 \mathrm{~b}$ Studien in der Impfstoffentwicklung erleichtern.

\section{Was soll ich tun? Beziehungsweise, was trägt das höhere Risiko?}

Wir sollten jetzt auf der Basis des derzeitigen Wissen vorgehen, uns nicht auf unsichere Verheißungen verlassen, aber jederzeit auch bereit sein, die Lage neu zu bewerten und Entscheidungen zu ändern. Ziel wäre, bildlich gesprochen, das Überleben des Indianerstammes. Der mühsame Weg in das Winterquartier wäre die fraktionierte Durchseuchung der Bevölkerung mit SARS-CoV-2, dem Wildvirus. Entsprechend der drei geforderten, hochkalibrigen, von einander unabhängigen Modellierungen, müsste ein System entwickelt werden, vermutlich ähnlich wie es von Ferguson et al. [9] schon skizziert worden ist, nach dem über zeitliche Episoden die NPI nach einem detaillierten Stufenplan gelockert und wieder angezogen werden. Möglichst bis knapp unterhalb der maximalen Auslastung der Krankenhäuser sollte die Durchseuchung zugelassen werden. Ziel wäre es die Gesamtzeit über das Jahr, in der die Maßnahmen "angeschaltet" sind, möglichst gering zu halten, um der Wirtschaft möglichst freien Lauf zu lassen. Deutlich unter $50 \%$ der Zeit sollte da in Anbetracht der Bettenkapazität in Deutschland zu schaffen sein. Wie das Feintuning im Detail aussehen könnte, wäre noch festzulegen, könnte aber je nach Bundesland in einer Art Mosaik in Deutschland in der zeitlichen und örtlichen Reihenfolge unterschiedlich sein.

Die größte Herausforderung wird das Feintuning der NPI unter Beibehaltung der Konstanten - Isolierung, Quarantäne und Schutz der vulnerablen Gruppen - werden. Die Schließungen von Kitas, Schulen und Universitäten müssen differenzieller betrachtet werden [11]. Hier könnte man sich weitere Untergruppen vorstellen, wie zum Beispiel Kinder unter dem Selbstversorgungsalter wegen Bindung berufstätiger Eltern zuhause. Allerdings haben Kinder bei SARS-CoV-2 nicht die zentrale Rolle für die Verbreitung wie bei Influenza; Jugendliche und junge Erwachsene schon eher wegen des hochdiversen und frequenten Kontaktmusters. Zum Beispiel könnte man eine Öffnung der Kitas und Grundschulen eher zulassen als die der weiterführenden Schulen und Universitäten.

Die stärkste Wirkung haben die generellen Kontaktbeschränkungen, wie sie über die Allgemeinverfügungen stufenweise eingeführt wurden $[1,9]$. Hier wären eine genaue Aufschlüsselung und mehr Informationen in Sachen Impact wünschenswert [13]. Auf private Veranstaltungen zu verzichten wäre das eine, aber auf berufsbedingte das andere. Man sollte sich an der Produktion orientieren. Die Einschränkungen in etwa in umgekehrter Reihenfolge aufzuheben, wie sie vor 4 Wochen sukzessive eingeführt wurden, könnte eine Richtschnur sein. Ebenso schwer in seinen downstreamEffekten abzuschätzen sind Mobilitäts-/ Reisebeschränkungen innerhalb Europas, die dennoch die Lieferketten nicht unterbrechen sollten. Ob der private Reiseverkehr innerhalb der EU und Anrainer zu begrenzen ist, ist eine schwierige Frage, die anderweitig zu diskutieren ist. Im Verlauf der Pandemie wären aber Bevölkerungsverschiebungen hin zu Ländern denkbar, die eine bessere Versorgung sicherstellen können. Dies wäre ein ernstes Problem, denn es sollte klar sein, dass die einheimische Bevölkerung den Vorrang und das Anrecht hat, in seinem eigenen Staatswesen versorgt zu werden; die "golden hour" eines jeden Staatswesens. Die bisherige Kooperation und das Mitdenken der Bürger hierzulande ist extrem lobenswert und 
hat viele Kontaktermittlungen der Gesundheitsämter erfolgreich sein und mit limitierten Maßnahmen entgegnen lassen. Das muss an dieser Stelle anerkannt werden.

Bei den Lockerungen müssen allerdings Puffer eingebaut werden, die schnell zu aktivieren gingen, wie zum Beispiel eine Unterstützung durch Sanitätseinheiten der Bundeswehr, denn Lockerungen betreffen komplexe Systeme, die fortlaufend die Tendenz haben, in chaotische Systeme zu kippen. Mit derartigen Absicherungen würden sich auch die Verantwortlichen leichter tun, Entscheidungen zur Lockerung zu treffen, nämlich wenn die Folgen abfangbar wären. Bei populationsbezogen mehr Betten in Deutschland als in Großbritannien, könnten die Phasen der bewegungseinschränkenden Maßnahmen um einiges kürzer werden, aber durch die doch erhebliche absolute Gesamtdauer wäre immer noch ein sehr hoher Preis zu zahlen.

\section{Was darf ich hoffen? Beziehungs- weise, worauf sollte man sich derzeit nicht verlassen?}

Auch wenn vor zwei Wochen, nach der kürzesten Zeit in der Medizingeschichte zwischen Publikation der genetischen Sequenz von SARS-CoV-2 am 9. Januar und dem Start der klinischen Entwicklung, die ersten Personen mit einem RNAImpfstoff der Firma Moderna in Boston geimpft wurden, illustriert die Schilderung von Etheridge [8] eindrücklich, wie durch limitierte Daten, Druck zu handeln und Fehleinschätzungen das Impfprogramm gegen ein neues Grippevirus schnell schwere unerwünschte Reaktionen bei Impflingen und somit einen erheblichen Kollateralschaden verursachte. Das präklinische safety Signal einer „enhanced“ Hepatitis bei Frettchen [5] gilt es nicht zu übersehen. Alle derzeitigen Impfstoffkandidaten setzen bisher laut CEPI auf das Spikeprotein als Impfantigen (https://cepi.net). In Anbetracht dessen sollte man sich immer wieder die bisherigen positiven Nachrichten vergegenwärtigen, nämlich die niedrige Hospitalisierungs- und Komplikationsraten bei Kindern und der jüngeren bis mitt- leren Altersgruppen, insbesondere Menschen ohne persönliche Risikofaktoren. So sehr sich jeder einen zeitnahen Erfolg der Impfstoffentwicklung wünscht und auch ein „magic bullet“ möglich ist, sollte man nicht alleinig darauf setzen und unabhängig davon den Weg der dosierten Durchseuchung der Bevölkerung gehen. Wir müssen uns darauf einstellen, das „Winterlager“ ohne einen ausreichend getesteten und ausreichend verfügbaren Impfstoff erreichen zu müssen, auch wenn die Entwicklungszeiten der Impfstoffe sich drastisch verkürzen und parallel dazu bereits die Produktionskapazitäten ausgeweitet werden.

\section{Was ist der Mensch? Beziehungs- weise, was kann/muss er verantworten?}

Die sich auftuenden ethischen Fragen sind umfangreich und Zielkonflikte sind vorprogrammiert. Die exekutive Entscheidungsmacht bei Fragen der Gesundheit liegt bei den Bundesländern. Unter geordneten Rahmenbedingungen, wie zum Beispiel ausreichend verfügbare Schutzausrüstung, sollten die Bundesländer nach Maßgabe eines Expertenkomittees in einer Art örtlichen und zeitlichen Mosaik die Lockerungen vornehmen, beginnend mit Bundesländern, die momentan weniger belastet sind. Je nach Belastungszahlen, Vorerfahrungen, unterschiedlichen Sichtweisen und Befindlichkeiten sind hier aber erhebliche Spannungen zu erwarten. Das System nicht ins Chaos gleiten zu lassen, bleibt die Herausforderung und der zentrale Balanceakt. Länder wie Deutschland, die die Gnade hatten, von einem ersten Chaos verschont zu bleiben, sollten unmittelbar mit dem hier Beschriebenen vorangehen, denn das verfügbare Zeitfenster bis November 2020 ist kurz. Und wie gesagt, ein nur teilweise getesteter Impfstoff wäre für die jungen und von SARS-CoV-2 wenig tangierten Gruppen ein unverhältnismäßiges Risiko.

An die Adresse der Kritiker und Bedenkenträger ist jetzt schon zu sagen, dass wir mit dem in dieser Arbeit Geschildertem vor der schwierigsten Aufgabe in dieser Pandemie stehen, nämlich die Durchseuchung fraktioniert zuzulas- sen und damit Tote in Kauf nehmen zu müssen. Das sind Entscheidungen, die jeden erschaudern lassen, die aber unausweichlich sind, wenn wir nicht noch größere Opferzahlen in einer übergroßen 2. Welle in Kauf nehmen wollen. Auf Populationsebene haben solche Entscheidungen nochmals größere Bedeutung als in der individuellen Triageentscheidung. Respekt und Ehrfurcht vor dieser übermenschlichen Aufgabe ist dringend geboten. Sich moralisch über andere, mit dem Thema ringende, Personen zu erheben, verbietet sich von selbst. Auch der politische und parteipolitische Wettstreit muss hier zurückstehen.

Bei den Einschränkungen des öffentlichen Lebens kam die Politik und Verwaltung schnell in den "flow“, ob das in umgekehrter Richtung auch so funktionieren und in Anbetracht der zu erwartenden Todesfälle auch so sein wird, wage ich zu bezweifeln. Öffentliche Gesundheit wird nicht nur von Ärzten entschieden, sondern auch von der Politik und Ministerien. Was jetzt gefragt ist, ist die weise Vorausschau und das couragierte Handeln von Führungspersonen, die dafür über die notwenige Kompetenz und Weitsicht verfügen müssen und auch Kurs halten können, wenn der Wind ins Gesicht bläst. Schnelles, proaktives Denken und die situativ angepasste, notwendige Flexibilität sind zu verlangen. Eine Kette ist nur so stark wie sein schwächstes Glied, ob national, regional oder international. Jetzt ist echtes, authentisches „Leadership“ gefragt. Der Philosoph Immanuel Kant kann uns dafür den Weg weisen. Lassen Sie uns ihm folgen, damit wir vor der Geschichte bestehen können.

Wirkliches Wissen besteht in der Macht und dem Vermögen, Tatsachen zu erkennen und Verknüpfungen zwischen Tatsachen herstellen zu können. Wissen ist ein Fürwahrhalten aus einem Erkenntnisgrund (Kant).

\section{Schlussfolgerungen}

Bei allem Respekt vor der Komplexität der pandemischen Lage sollte dennoch couragiert gehandelt werden und jetzt schon die Überlegungen für die nächsten Wochen, Monate und Jahre 
angestellt werden. Ungeachtet der Verlockungen durch Verheißungen aus der Impfstoffentwicklung sollte jedes Gesundheitswesen, allen voran die bisher vom Chaos verschont gebliebenen vorwärts schreiten, ohne sich auf zeitnahe Erfolge und Verfügbarkeit von Impfstoffen und kausalen Therapeutika zu verlassen. Die Zeit bis zur nächsten saisonalen Bettenknappheit ab Herbst 2020 drängt. Mehrere Modellierungen, wenn auch mit Restunsicherheiten, sollten unabhängig voneinander in Auftrag gegeben und dann durch ein möglichst hierarchie-heterogenes Gremium („post-westphalian“) beurteilt werden und die entsprechenden Triggerpunkte und Stellgrößen beschlossen werden. Auch unsere Vorfahren haben die sogenannte Russische Grippe 1889/1890, durch ein Coronavirus verursacht, überlebt und das „Winterlager" erreicht. Auf Modellierungen, epikritische Beurteilung der Impfstoffentwicklung und genuines Leadership kommt es jetzt an.

\section{Fazit für die Praxis}

- Die Planungen und Ausdifferenzierung der Maßnahmen für die nächsten Monate und Jahre müssen jetzt schnell vorangetrieben werden. Mehrere, voneinander unabhängige Modellierungen sind für die Überlegungen die notwendige Grundlage. Das Imperial College, London, sollte unter anderem von der Bundesregierung dazu beauftragt werden.

- Die fraktionierte Durchseuchung sollte unmittelbar nach Einsetzung der ersten Bremswirkung weiter verfolgt werden, um eine übergroße 2. Welle zu vermeiden.

- Sich auf die Verfügbarkeit eines nur marginal getesteten Impfstoffes zu verlassen, wäre fahrlässig. Wenn überhaupt, dann kommt ein noch unsicherer Kandidatenimpfstoff allenfalls für Personen mit einem erhöhten persönlichen Risiko in Frage (vulnerable Gruppen).

- Die Philosophie von Kant ist für volatile, ungewisse, komplexe und uneindeutige Lagen eine zeitlose Richtschnur, auch für die notwendigen, persönlichen Wandlungsprozes- se in Sachen pandemie-adäquaten Gebarens und Managements.

\section{Korrespondenzadresse}

PD Dr. med. habil. Josef Weigl, MTropPaed, DTMH, DipEPP

Gesundheitsamt Plön, Schleswig-Holstein Hamburger Str. 17/18, 24306 Plön, Deutschland josef.weigl@kreis-ploen.de

Widmung. Ich möchte diese Arbeit meiner vormaligen pädiatrischen Kollegin Lucia widmen, deren Mann vor zwei Wochen einen thorakalen Vernichtungsschmerz verspürte und daraufhin von zwei kardiologischen Notaufnahmen wieder nach Hause geschickt wurde vor dem Hintergrund, die Kapazitäten möglichst für Pandemiepatienten bereitzuhalten. Erst mit über 10 Tagen Verzögerung wurde dann durch Veranlassung der Hausärztin eine bildgebende Diagnostik durchgeführt und der Patient notfallmäßig wegen einer Aortendissektion operiert. Dass es in der Pandemie auch andere, lebensbedrohliche Erkrankungen gibt, die zudem dann schon eingetreten sind, darf nicht wegen der Pandemievorbereitung und Kapazitätenvorhaltung vernachlässigt werden.

\section{Einhaltung ethischer Richtlinien}

Interessenkonflikt. J. Weigl gibt an, dass kein Interessenkonflikt besteht.

Dieser Beitrag enthält keine Untersuchungen an Menschen oder Tieren oder personenbezogene Daten.

\section{Literatur}

1. Anderson RM, Heesterbeek $H$, Klinkenberg D, Hollingsworth DT (2020) How will country-based mitigation measures influence the course of the COVID-19 epidemic? Lancet. https://doi.org/10. 1016/S0140-6736(20)30567-5

2. Anderson RM, Fraser C, Ghani AC et al (2004) Epidemiology, transmission dynamics and control of SARS: the 2002-2003 epidemic. Philos Trans R Soc Lond B Biol Sci 359:1091-1105

3. An der Heiden M, Buchholz U (2020) Modellierung von Beispielszenarien der SARSCoV-2-Epidemie 2020 in Deutschland. https:// www.rki.de/DE/Content/InfAZ/N/Neuartiges_ Coronavirus/Modellierung_Deutschland.pdf? blob=publicationFile. Zugegriffen: 24. März 2020. https://doi.org/10.25646/6571.2

4. Covey SMR (2006) The speed of trust - the one thing that changes everything. Free Press, New York

5. Czub M, Weingartl H, Czub S et al (2005) Evaluation of modified vaccinia virus Ankara based recombinant SARS vaccine in ferrets. Vaccine 23:2273-2279

6. D'Alessandro DF, Owens M (2008) Executive warfare-10 rules of engagement for winning your war for success. McGraw-Hill, New York

7. Edwards K, Hanquet G, Black Set al (2019) Meeting report narcolepsy and pandemic influenza vaccination: What we know and what we need to know before the next pandemic? A report from the 2ndIABS meeting. Biologicals 60:1-7

8. Etheridge EW (1992) 1976. In: Sentinel for health-a history of the Centers for Disease Control. University of California Press, Berkley, S247-267

9. Ferguson NM, Laydon D, Nedjati-GilaniG (2020) Impact of non-pharmaceutical interventions (NPIs) to reduce COVID-19 mortality and healthcare demand https://doi.org/10.25561/77482 (Imperial College COVID-19Response Team)

10. Fidler D (2004) SARS, governance and the globalization of disease. Palgrave MacMillan, Basingstoke

11. Fumanelli L, Ajelli M, Merer S, Ferguson NM, Cauchemez S (2016) Model-based comprehensive analysis of school closure policies for mitigating influenza epidemics and pandemics. PLoS Comput Biol 12:e1004681. https://doi.org/10.1371/ journal.pcbi.1004681

12. Guan W, Ni Z, Hu Y et al (2020) Clinical characteristics of coronavirus disease 2019 in China. N Engl J Med. https://doi.org/10.1056/NEJMoa2002032

13. Haw DJ, Cummings DAT, Lessler J et al (2019) Differential mobility and local variation in infection attack rate. PLoS Comput Biol 15:e1006600. https://doi.org/10.1371/journal.pcbi.1006600

14. Höffe O (2000) Immanuel Kant. Becksche Reihe, Bd. 506: Denker.C.H. Beck, München

15. Menachery VD, Yount BL Jr, Debbink Ket al (2015) SARS-like cluster of circulating bat coronavirus pose threat for human emergence. Nat Med 21:1508-1513

16. Niquet B (2007) Kant für Manager. Campus, Frankfurt am Main

17. Weigl J (2020) Das VUCA plus der Tuberkulose in Deutschland und die Notwendigkeit einer Strategie 2.0. Präv Gesundheitsf. https://doi.org/ 10.1007/s11553-020-00778-w

18. Weigl J (2020) Betrachtung der Pandemie Phase 3 - "Mitigation" - vom Endpunkt Hospitalisation her. Präv Gesundheitsf. https://doi.org/10.1007/ s11553-020-00771-3

19. Weigl JAI, Puppe W, Meyer CU et al (2007) Ten years active-year round surveillance of up to 19 respiratory pathogens in children. Eur J Pediatr 166:957-966

20. Weigl J, Forster J, Berner R et al (2003) Virale Atemwegsinfektionen mit saisonaler Häufung bei Kindern. Eine Übersicht mit Schwerpunkt auf Daten aus Deutschland. Bundesgesundheitsblatt 46:9-19

21. Wrapp D, Wang N, Corbett KS et al (2020) Cryo-EM structure of the 2019-nCoV spike in the prefusion conformation. Science 367:1260-1263 